\title{
IntermationalJoumal of ArtsandHumanities(IJAH)
}

\section{Ethiopia}

Vol. 7 (2), S/No 25, APRIL, 2018: 54-61

ISSN: 2225-8590 (Print) ISSN 2227-5452 (Online)

DOI: http://dx.doi.org/10.4314/ijah.v7i2.8

\section{Repenser la Sorcellerie dans les Romans Antillais: Une étude Sociocritique de Moi, Tituba Sorcière... Noire de Salem de Maryse Condé, Chair Piment de Gisèle Pineau et Hadriana dans tous mes Rêves de René Depestre}

\author{
Obidiegwu, Vincent Nnaemeka \\ Department of Foreign Languages and Literary Studies \\ University of Nigeria, Nsukka \\ Phone: +2348065679896 \\ E-mail: vincentobidiegwu@gmail.com \\ Ugwu, Evaristus Nkemdilim \\ Department of Foreign Languages and Literary Studies \\ University of Nigeria, Nsukka \\ Phone: +2348137154600 \\ E-mail: ugwuevaristusn@yahoo.com
}

\begin{abstract}
Sorcery is a subject that always creates heated debate in our contemporary society. That's why we aim to examine sorcery within the specific context of Caribbean writer's literary creations. Therefore, this study looks at witch crafting as one of the major themes that continue to occupy a major place in the Antillean literary discourse. It seems to us that irrespective of the presence of this theme, very rare are literary critics especially from Nigeria that is based on sorcery. That forms our reason of venturing into these literary works to unveil and show how witch crafting is represented. What are the reasons behind this? The overall assessment is it negative or positive? What is the way forward? These are basic questions that this study tries to resolve under social critic approach.
\end{abstract}

Key Words: Rethink, witch crafting, Antillean, social critic

\section{Résumé}

La sorcellerie est toujours dans notre société contemporaine un sujet qui suscite des polémiques. C'est ainsi que nous allons l'examiner dans le contexte particulier des créations littéraires des écrivains antillais. Donc, cette étude se porte sur une réflexion sur la sorcellerie comme l'un des sujets qui continue à occuper une place prépondérante dans les discours littéraires antillais. Il nous semble aussi que très rares sont des critiques littéraires qui se porte sur la sorcellerie. C'est ainsi que nous voulons s'aventurier dans ces œuvres littéraires pour démontrer dans quelle mesure la sorcellerie y est représentée. Pour quelle raisons ce sujet reste au cœur de débats dans le monde littéraire antillais ? Le 
bilan est-il négatif ou positif? Quels sont les perspectives de l'avenir ? Ce sont des questions de base dont cette communication cherche à résoudre sous l'approche sociocritique.

Mots clés: Repenser, sorcellerie, antillais, approche sociocritique

\section{Introduction}

La littérature dans sa nature engagée reste un miroir d'une société donnée dans une époque donnée. Elle est mandatée à projeter une société de référence telle qu'elle est en exposant bien sûr ses atouts et ses problèmes tant social que politique voire économique dont elle tentera de condamner et chercher des solutions propices pour le bonheur de cette société en particulier et le monde en général. Unimna (2008) a proprement dit que: «la littérature antillaise est une littérature qui est plus que l'imagination. Elle se veut la chronique, l'histoire d'un peuple qui se voit toujours cloué sous l'éteignoir d'un système socio-politico-économique qui le broie » (5). Par conséquence, chacun des écrivains antillais contemporaines essaient de poétiser, à leurs manières et selon le fonctionnement de leurs imaginations créatrices, les conditions misérables dans lesquelles ce peuple vit et l'implication du phénomène de la sorcellerie dans leurs milieu social. Ainsi, chez les romancières antillaises telles Maryse Condé et Gisèle Pineau qui viennent de souche Guadeloupéenne, René Depestre un Haïtien, qui est un de figure remarquable dans la littérature haïtien, nous avons constaté qu'ils évoquent respectivement dans leurs œuvres romanesques la question de la sorcellerie qui se révèlent comme un veritable tabou chez des individus dans le monde contemporaine. C'est ainsi que nous voulons s'aventurier dans cette étude dans la perspective sociocritique pour démontrer dans quelle mesure la sorcellerie y est représentée à travers les trois œuvres retenus tels Moi, Tituba Sorcière. . . Noire de Salem de Maryse Condé, Chair Piment de Gisèle Pineau et Hadriana dans tous mes rêves de René Depestre? On se demande si l'inscription de ce thème clé dans la littérature antillaise est une chose gratuite ou y a-t-il des messages cachés derrière ce thème qui pouvaient améliorer la vie de l'homme antillais? Donc, dans cette communication, nous allons essayer de jeter le flambeau sur la sorcellerie en tant que l'un des thèmes principaux dans le roman antillais.

A vrai dire, bon nombre de travaux critiques sont produits sur plusieurs thématiques tels que : la discrimination, l'oppression, l'exploitation, l'exil, la crise d'identitaire pour ne mentionner que ceuxlà mais il nous semble que très peu sont des travaux critiques produits sur la sorcellerie comme thème surtout auprès des critiques nigérians dans les œuvres mentionnée. Voila la raison d'être de cette article qui nous ouvre l'occasion précieuse de pénétrer dans le corpus littéraires dans les buts de découvrir comment ce theme est représenté, son image est-il négatif ou positif? Quelle est la perspective d'avenir laissée par nos écrivains? A ce stade, il convient d'abord de donnée des petits résumés de ces trois romans qui forment les toiles de fond de cette étude.

\section{Résumé des Euvres Choisies}

Moi, Tituba Sorcière... Noire de Salem (1986) est une œuvre littéraire de Maryse Condé qui projette le récit d'une femme esclave, Tituba qui à travers plusieurs espaces : Barbade, Bridgetown, Boston, Salem et Ipswich est devenue une victime d'oppression, discriminations, exploitation à cette époque de l'esclavage. Rassemblée autour d'elle-même l'image d'une guérisseuse, une écologiste, une femme tolérante et courageuse, Tituba, l'héroïne narratrice finira par devenir enfin, une figure de résistance et la voix de sans voix non juste pour ses compatriotes noirs mais pour tout homme opprimée.

Chair Piment (1996) est une œuvre littéraire de Gisèle Pineau qui met en relief de sa part, le récit d'un certain Monsieur Melchoir dont son aventure maritale échouée a plongé toute la famille dans le chaos. Mina Monterio, la fille de Melchoir frappée par beaucoup de malheurs à Piment sera exilée en France en cherchant en vain de s'y échapper. Mais grâce à son retour à Piment, le pays natals, après plusieurs années de souffrance, elle découvrira et fera la paix avec Suzon Mignard le cerveau derrière tous ces malheurs qui ont frappé sa famille. 
Hadriana dans tous mes rêves (1988) est un récit consacrée ò une jeune femme, Hadriana dont sa mort inattendue au jour de son mariage a soulevé tout un tas de problèmes qui confrontent la société jacmelienne, l'espace du roman. Les circonstances qui entourent cette mort et la pratique de la sorcellerie sont des sujets épineux devant le peuple jacmelien qui saisit cette occasion pour discuter les problèmes de leur société. Alors passons-nous au corpus littéraire.

\section{La Sorcellerie dans les Romans Antillais}

Dans le premier roman, Moi, Tituba Sorcière... Noire de Salem, la rencontre entre l'héroïne narratrice Tituba et John Indien nous présente à nu la position de Condé sur la sorcellerie en tant qu'une pratique, ou métier dans la société humaine. Maryse Condé à travers Tituba l'héroïne narratrice s'interroge sur la justification de ce mot :

Qu'est-ce qu'une sorcière? Je m'apercevais que dans sa bouche, le mot était entaché d'opprobre. Comment cela? Comment? La faculté de communiquer avec les invisibles, de garder un lien constant avec les disparus, de soigner, de guérir n'est-elle pas une grâce supérieure de nature à inspirer le respecte, l'admiration et la gratitude? En conséquence, la sorcière, si on veut nommer ainsi, elle qui possède cette grâce ne devrait-elle pas être choyée et révérée au lieu d'être crainte ? (34).

En effet, la sorcellerie est une pratique courante dans la société afro-antillaise et également représentée dans beaucoup d'œuvres littéraires appartenant à ces mondes. Il faut souligner que, la sorcellerie est une véritable force capable de faire du bien ou du mal selon son application. Elle a comme d'autres connaissances humaines avec ses côtés positif et négatif. Bien sûr que dans le contexte actuel, cette notion est mal vu surtout dans le monde chrétien qui la considère comme une affaire diabolique, Condé semble voulait redéfinir la sorcellerie et rebâtir son image bien bafoué à cause de l'ignorance et la naïveté. Effectivement, il est indubitable que Tituba met en valeur le rôle bienfaisant joué par la sorcière dans la société en disant que " la "sorcière" si nous devons employer ce mot, corrige, redresse, console, guérit... » (152). Il est clair que la représentation de ce mot "la sorcière" n'est pas tout à fait gratuit dans les œuvres afro-antillaise mais pour un appel d'évaluation et réévaluation de ce secteur et son rôle majeur dans la société. Comme le remarque Sullivan (2017) : « dans Moi, Tituba sorcière... Maryse Condé explore ces thèmes tout en exposant des enjeux sociaux, raciaux et culturels rattachés à la sorcellerie. En s'appuyant sur la représentation historique de la sorcière, soit celle de la victime en marge de la société,... » (72).

Par ailleurs, dans Les soleils des indépendances (1970) d'Ahmadou Kourouma où le narrateur s'est remémoré que : «Fama n'avait pas écouté les paroles prophétiques du grand sorcier, Balla, lors du départ de Togobala. Effectivement, cela lui paraissait maintenant incroyable et c'est pourtant vrai » (176). Ainsi, dans cette œuvre retentissante de Kourouma, nous voyons comment le héros, Fama pourrait échapper le malheur qui lui a frappé à la capitale s'il avait écouté l'avertissement du sorcier, Bala depuis au village après les obsèques de son oncle, Lacina.

Chez Maryse Condé, Tituba l'héroïne s'est servie de cette connaissance pour soigner et guérir sa maîtresse Elizabeth Paris, la femme de son maître après plusieurs jours de maladie. Comme le témoigné par l'héroïne narratrice :

Cette nuit-là, je décidai d'avoir recours à mon talent... Je décidai d'user de subterfuges. Un arbre dont le feuillage virait au rouge fit de fromager. Des feuilles de houx acérées et luisantes, remplacèrent les herbes de Guinée... Au matin, les couleurs revinrent aux joues de Maitresse Elizabeth Paris. Elle réclama un peu d'eau à boire. Vers le milieu de la journée, elle parvint à s'alimenter. Le soir venu, elle s'endormit comme un nouveau-né. Trois jours plus tard, elle 
m'adressait un sourire frileux comme le soleil à travers les lucarnes: merci, Tituba! Tu m'as sauvé la vie! (75-76).

Sullivan (2016) souligne ce point de vue dans son article lorsqu'elle dit qu' " elle met en doute les termes qui sont employés pour la décrire et réfléchit de manière critique le rôle qu'elle joue en société, comme guérisseuse, conseillère et amie des villageois » (17). Ainsi, il faut souligner que Tituba représente plus ou moins la position de Condé sur ce sujet sensible et tranchant. Condé maintient que pour elle, il faut s'en servir de cette science pour améliorer la condition humaine. Comme le remarque l'héroïne résistant la tentation de faire du mal avec cette connaissance de la sorcellerie en prison: « Je ne peux pas faire ce que ton cœur n'ose même pas formuler. Celle qui m'a communiquer sa science m'a appris à guérir, à apaiser plus qu'à faire du tort. Une fois où, comme toi, je rêvais du pire, elle m'a mise en garde : ne deviens pas comme eux qui savent que faire le mal» (109).

A ce stade, il faut que nous passions au deuxième corpus littéraire: Chair Piment de Gisèle Pineau. Pour voir comment Gisèle Pineau, dans son imagination créatrice a pu représenter la sorcellerie comme un sujet très épineux dans le roman antillais. Dans cette œuvre, Pineau a essayé de mettre en relief deux applications de cette pratique ; l'une pour la guérison et l'autre pour la vengeance. Deux personnages sont impliqués notamment Victor, un français et Suzon Mignard, une Guadeloupéenne. Notons que dans un hôpital psychiatre d'Esquirol en France, Le jeune Victor se trouve pour essayer de chercher la solution médicale à sa santé qui ne fait que se dégrader. En fait, à travers le témoignage du narrateur, Victor dit que:

Je suis venu chercher refuge, me sauver de moi-même qui suis mon pire ennemi se disait-il, apparemment serein, tableau classique du mélancolique au bord du suicide. Une fois de plus, il avait touché le fond, avait voulu en finir avec sa propre vie. Je suis raté. Ma mère m'a raté. Tout a fait depuis ma naissance. Les auraient pu être heureux si je n'étais pas né. C'était un bel homme Edmond. Ma mère l'aimait bien. Et puis il se pend, au lustre, au milieu du salon et... (106).

Ceci démontre l'état de ce jeune français chez lui en France avec tant d'infrastructures pour rendre la vie plus belle, mais pour Victor, il est fatigué pour vivre, pour lui, le suicide devient une option comme le cas de son défunt père. Mais grâce à sa rencontre dans cet hôpital avec Bénédicte, une antillaise, l'équation sera changée.

Voici le conseil de Bénédicte:

Tu peux être sauvé... il faut me croire ! Je les vois comme je te vois. Trois démons qui te laissent pas en paix depuis ta naissance. Souviens-toi de Jésuschrist! Les démons nous font la guerre, tu entends! Mais vous les voyez donc pas... N'oublie pas! Faut que t'ailles te faire désenvoûter en Guadeloupe, Victor. (107-110).

Ainsi, grâce à son voyage en Guadeloupe et sa rencontre avec un homme appelé Monsieur Vérité (M. Vérité), réputé de connaissance au domaine de la sorcellerie, Victor sera guéri. A ce propos, Victor dit que : «depuis qu'il était en Guadeloupe, il avait réussi à vaincre quelques vieilles peurs... il est maintenant sûr que M. Vérité avait déverrouillé une porte-comme disait l'un de ses psychiatres-qui ouvrait sur le chemin de la guérison » (351). Très spectaculairement, nous voyons ici que pendant que les Antillais voyaient leurs îles natales comme des enfers les plus cruels du monde démontrés par plusieurs œuvres littéraires antillaises, certains aventuriers tels que Victor un blanc ont fini par trouver leurs saluts aux Antilles notamment à Guadeloupe. Bouchard (2004) dit à cet égard que : "Victor, homme blanc habituellement privé de volonté, décidera, sous les conseils de Bénédicte, d'aller se faire désenvouter en Guadeloupe. Homme blanc, européen, catholique, il délaissera la médecine 
moderne et choisira la médecine traditionnelle antillaise » (62). Ceci dit, on se demande si Gisèle Pineau, veut-elle nous dire qu'il existe tant d'opportunités à célébrer aux Antilles ? Voulait-elle nous dire qu'aucun pays est vide car chaque pays est toujours doté des atouts qui peuvent permettent son décollage socio-économique et politique ? Alors pour démontrer la capacité et les deux côtés en ce qui concerne l'application de la science sorcière ou la sorcellerie, Pineau dans cette production littéraire est allée très loin pour mettre en valeur le sort et la réaction d'une certaine Suzon Mignard dont son aventure maritale avec monsieur Melchoir caractérisée par beaucoup de promis et d'échecs qui l'a poussé à appliquer la science de la sorcellerie négativement.

Mais avant de commenter sur la réaction de cette Suzon dont la sorcellerie est impliquée, faisons un peu de la marche arrière sur la cause principale représentée dans le roman car il n'y a pas la fumée sans feu. En effet, le narrateur nous fait entendre que :

Melchoir lui promit la lune et bien d'autres planètes. Pour lui, Suzon retira, dans la noirceur amie, sa culotte blanche immaculée. Tremblant, Melchoir glissa sa main entre les cuisses de l'aimée. Le pressa, caressa, Mignone tant et plus avant de fourrer son bâton d'homme dans le corps qui roulait et tanguait déjà .... Elle l'autorisa à déchirer le voile de sa virginité. Elle saigna pour lui, rien que pour lui... (162).

Alors, c'est le début d'une rencontre entre deux amis qui finira dans la tragédie. L'aventure maritale entre Suzon Mignard et Monsieur Melchoir bien débuté comme le narrateur à travers ses yeux scrutateurs nous a mis en relief plus haut va malheureusement tourner mal. Selon le témoignage du narrateur : «Suzon avait connu l'abandon, la pétrification, l'attente et la brûlure de la haine. Imaginez cette forteresse de patience en ruine » (174). C'est dans cette circonstance que Pineau veut démontre l'application négative ou bien l'application destructive de la sorcellerie biais les réactions de cette Suzon qui s'est vengée contre la famille Melchoir qui l'a ruiné la vie. De ce fait, Rebeix (2017) dit qu' « en ce sens, Suzon Mignard, l'un des personnages centraux de Chair Piment, incarne de façon hyperbolique la femme délaissée par son premier amour» (166). A cet égard, le narrateur nous fait comprendre que Suzon Mignard en préparant pour cette lutte est devenue "la sorcière qui visita cinquante-quatre " gadezafé » et dix sorciers afin de voir triompher l'amour. L'immoral qui convoqua les esprits mauvais des eaux, bloqua les freins d'un camion Titan, héla la folie, la foudre et le feu ...» (174). Il faut dire qu'à travers la sorcellerie, Suzon Mignard a réussi à éliminer les deux premières femmes de monsieur Melchoir considérées par elle comme des obstacles par rapport au mariage prévu entre elle et Melchoir. En formant un très fort réseau de sorciers pour sa mission de vengeance. Suzon Mignard est devenu incontournable. A partir de là, le narrateur décrit, comment les deux femmes de Melchoir (Marie-perle et Médée) ont jeté les éponges biais la science de sorcellerie. Ainsi, Gisèle Pineau nous en valeur le palmarès de ces deux sorciers qui ont perpétré ce crime haineux :

Tonnere-de-Dieu était de la foudre et des éclairs. D'un seul coup d'œil, il pouvait faire le ciel viré au noir. Il avait jurait-il, vu trois fois l'Apocalypse. Il déchaîna, pour Suzon, les eaux de la rivière Goyave qui emportèrent Marie-Perle. Apolythe Trident naquit noir de peau et mourut plus blanc que lait. Sorcier de tradition, il aimait la modernité. La mécanique était son domaine réservé. Il provoquait les accidents sans quitter la touffe de son officine, parlait aux freins, aux soupapes et aux carburateurs. Il endormait les chauffeurs au volant des automobiles, faisait apparaître des obstacles imaginaires et traçait des routes qui menaient au néant. Suzon le sollicita quand elle voulait finir avec Médee... (176). 
Ainsi, il est à préciser qu'à travers la vengeance de cette femme, Suzon contre ses ennemis, Pineau démontre davantage la capacité et le rôle de sorciers en nous donnant la liste de vingt sorciers très réputés d'origine antillaise et de leurs savoir- faire dans ce domaine. Selon Pineau :

Ce n'était pas comme maintenant. Autrefois, les sorciers savaient y faire. Gens de métier, héritiers d'un savoir millénaire, ils possédaient leur art et maniaient sans trembler les feuillages et les racines. Les poussières d'étoiles et le limon des eaux troubles. Ils commandaient les éléments au ciel et sur la terre. Ils travaillent avec la lune et le soleil. Parlaient aux esprits défunts et lisaient la destinée dans la jaune d'un œuf couvé. Comptaient les enfants au ventre des vierges ... (175).

Donc, il nous semble que Gisèle Pineau à travers cette création littéraire veut démontrer la capacité de la sorcellerie étant un métier peut construire ou détruire selon son application.

Par ailleurs, il nous impose de jeter un regard sur le troisième roman : Hadriana dans tous mes rêves de René Depestre. Dans ce roman, nous avons toujours constaté que la sorcellerie sous de nombreuses formes se fait toujours un thème clé de cette œuvre classique. Comme le note Lucas (2002) "René Depestre exploite dans Hadriana de tous mes rêves (1988) le potentiel suggestif de la zombification vaudou pour en tirer une surréalité onirique compensatrice, et pour insuffler une force poétique à une réflexion sur la mort, mort dédramatisée » (194). En effet, il est clair que René Depestre à travers son œuvre, porte un regard percutant sur la sorcellerie en tant qu'une pratique pour détruire des êtres humains aux Antilles. Autrement dit, pour faire du mal. Ainsi, dans l'espace romanesque de Jacmel en Haïti, Balthazar Granchiré, un enfant adoptif du fameux sorcier Okil Okilon est l'une des personnages qui représente cette destruction à travers son métier de la sorcellerie. En fait, le narrateur nous fait comprendre que:

Balthazar Granchiré arriva à Jacmel pour la première fois en novembre 1936... il élut domicile sur l'un des fromagers de la place d'Armes. La nuit de son arrivée, il déflora pendant leur sommeil les jumelles Philisbourg et sœur Nathalie des Anges, l'une des religieuses de l'école Sainte-Rose-de-Lima...Il attendait l'obscurité pour se faufiler dans les chambres. Il se dissimulait sous les lits. Une fois sa proie endormie, il imprégnait l'atmosphère d'effluves aphrodisiaques. Quelques minutes après ... De superbes adolescentes, couchées vierges sous la protection du cocon familial, se réveillaient dans l'effroi, avec du sang partout sauvagement dépucelées... (28).

Ainsi, dans ce genre de situation où les écrivains antillais ne font que représenter la société antillaise telle qu'elle est, cependant, comme nous avons vu à travers toutes ces œuvres au sujet de sorcellerie, est-il un moyen de peindre en noir cette société ? Loin de cela, il faut souligner que l'écrivain en mettant à nu la situation sociopolitique et économique antillaise cherche toujours à solliciter l'attention des critiques de quatre coins de monde dont leurs contributions pourraient améliorer le sort de l'homme antillais. En effet, dans ce roman, on notera la mort d'Hadriana Siloé, la plus belle femme de Jacmel au jour de son mariage de noces comme une affaire de la sorcellerie et cela continue à y faire la polémique. Comme le précise Sergio (2005) «En effet le livre est centré sur les événements mystérieux qui cernent la mort d'Hadriana et la disparition de son corps. Pour l'un des protagonistes, Patrick Altamont, la mort d'Hadriana signifie un bouleversement total de sa vie » (6). C'est ainsi que les citoyens Jacmeliens pointent le doigt à ce sorcier dangereux, Balthazar Granchiré. Comme le dit le narrateur: " ... Le décès d'Hadriana n'était pas dû à une cause naturelle. On n'avait pas besoin du talent de Sherlock Holmes pour découvrir la piste qui conduisait à l'auteur du forfait. Celui-ci était signé Balthazar Granchiré »(49). En évoquant la mort de cette jeune femme comme celui de Monsieur Francis Sancher chez Maryse Condé dans Traversée de la mangrove, elle est devenue une occasion précieuse dont le peuple Jacmelien qui représente le peuple antillais utilise pour faire un 
bilan sur leur vie sociale. C'est pourquoi le narrateur dit: «en vérité, c'est toute la terre qui est humiliée lorsqu'une jeune fille de dix-neuf ans est foudroyée le soir de ses noces ! » (56). En grosso modo, le peuple Jacmelien fait face à plusieurs contextes où il s'interroge sur les activités de la sorcellerie dans leurs pays en guise d'avoir une révolution par rapport au usage de cet art incontournable.

\section{La Perspective de l'avenir}

Dans cet aspect, il convient de noter qu'en faisant la lumière sur la sorcellerie dans la société antillais contemporaine et en mettant l'emphase sur ce thème clé qui jalonne effectivement leurs productions littéraires, il nous semble que chez des écrivains antillais, pour écrire sur la sorcellerie ce n'est pas un hasard mais plutôt une démarche audacieuse qui cible développement positif de ce domaine. A cet égard, le fait qu'une écrivaine chevronnée comme Maryse Condé revendique farouchement cette pratique dont elle voyait son rendement immense, non juste dans la société antillaise mais dans le monde entier, nous dit que l'avenir de la sorcellerie tracé par la plupart des écrivains reste promettant mais il faut révolutionner cette pratique.

\section{Conclusion}

Dans l'ensemble de notre étude, nous avons tenté de suivre les personnages de Condé, de Pineau, de Depestre dans les aventures telles qu'elles sont présentées dans les corpus. Ce qui ressort de notre étude est que la pratique de la sorcellerie demeure un art très omniprésent dans le parcours de ces écrivains. Il est clair que certains individus l'utilise pour le bien-être humain comme nous avons remarqué chez Condé et Pineau tandis que certains font l'usage de cet art pour faire la vengeance ou bien de perpétuer de mal comme le cas de Suzon Mignard chez Pineau et Balthazar chez Depestre. Par ailleurs, la sorcellerie comme d'autres connaissances est dotée du côté positif et du côté négatif. Nous avons constaté que des écrivains sont d'avis que cette pratique pourrait aider les Antilles surtout au domaine de la science si c'est bien appliquer pour le bonheur de l'homme. Il est fort évident que les écrivains tentent de promouvoir ou bien valoriser la culture antillaise en faisant l'éloge d'aspect positif de la sorcellerie dans leurs œuvres romanesques. Il essaie de s'opposer à l'idée occidentale qui voie la culture antillaise à travers la sorcellerie comme négatif. Entant donnée que la culture est un facteur socioéconomique, les écrivains veulent montrer par leurs œuvres le bienfait de la sorcellerie, qui peuvent donner une bonne représentation de la société antillaise, attirant ainsi, des possibilités du tourisme médical.

Dans cette optique, il nous semble que cette démarche pour développer la société antillaise socialement, économiquement voire politiquement, les écrivains songent à une réévaluation de toutes valeurs culturelles antillaises dans le but de développer positivement certaines valeurs qui pouvaient catalyser le développement aux Antilles. Par conséquence, elles peuvent assurer la stabilité de l'homme antillais actuellement dans l'errance continue. Nous recommandons qu'il est pertinent de mettre en place une plate forme durable qui se chargera de la sorcellerie en tant qu'un domaine ou un métier qui existe dans la société, afin d'encourager la sorcellerie positive et de décourager le pratique néfaste de la sorcellerie. Donc, il faut songer à savoir comment cet art peut aider les antillaise et les pays en voie de développement surtout au niveau d'innovation au lieu de la condamnée entièrement. C'est cela le débat dont cette étude a essayé de soulever. Nous sommes persuadés qu'au contexte actuel de la sorcellerie, il y demeure obligatoirement les atouts qu'il faut exploiter.

\section{Cuvres Citées}

Bouchard, Catherine. Discours sociaux et aliénation dans trois romans de Gisèle Pineau, Diss. Mémoire de la maitrise, Université de Montréal, Aôut, 2004.

Depestre, René. Hadriana dans tous mes rêves. Paris: Gallimard, 1988. 
Kourouma, Ahmadou. Les Soleils des indépendances, Paris : Seuil, 1970.

Lucas, Rafael. «L'esthétique de la dégradation dans la littérature haïtienne », Revue de littérature comparée 2. 302. 2002. 191-211.

Maryse, Condé. Traversée de la mangrove. Paris: Mercure, 1989.

........, Condé. Moi, Tituba, sorcière noire de Salem. Paris: Mercure, 1986.

Pineau, Gisèle. Chair Piment. Paris: Mercure de France, 2002.

Rebeix, Stéphanie. «Du fantastique au réalisme merveilleux dans deux romans de Gisèle Pineau Chair Piment et Cent vies et des poussières ». GRELCEF. 9. 2017. 159-178.

Sergio, I. Lagman Jr. L'Importance du Vaudou dans Hadriana dans Tous Mes Rêves de René Depestre, Diss. Masters, The Florida State University College of Arts and Sciences, 2005.

Sullivan, Maryse. «Au ban de la société, à la frontière de l'Amérique: les sorcières et les marginaux dans Moi, Tituba sorcière ... de Maryse Condé », Voix Plurielles. 14.1. 2017.

........., Maryse. « Les femmes marginales de l'Histoire: les sorcières de Maryse Condé et de Nancy Huston » Chimères Vol XXXIII. 2016.

Unimna, Angrey. Le roman de Maryse Condé et les perspectives d'avenir des Antilles, Calabar: Optimist Press Nig. Coy. 2008. 202. 